\title{
Fibroblast Heterogeneity in Periodontium - a Review
}

\author{
Dr. A. Archana ${ }^{1}$, Dr. Venkata Srikanth ${ }^{2}$, Dr. Sasireka ${ }^{2}$, Dr. Bobby Kurien ${ }^{2, *}$, Dr. Ebenezer ${ }^{2}$ \\ ${ }^{1}$ Department of Periodontics, Madha Dental College, Chennai, India \\ ${ }^{2}$ Department of Periodontics, Adhiparasakthi Dental College, Chennai, India \\ Corresponding author: bobbds2000@gmail.com
}

Received April 28, 2014; Revised May 09, 2014; Accepted May 11, 2014

\begin{abstract}
Fibroblast heterogeneity applies to both phenotypic and functional properties exhibited by a fibroblast population within or across tissues (Mc Culloch et al) There is significant evidence that fibroblasts are heterogeneous with respect to functional properties, and that certain subpopulations of these cells may be clonally selected and expanded in diseased tissues. There are wide variations of gene expression and strikingly different responses to extracellular signals among different fibroblast populations. This has prompted a large number of in vitro studies which suggest that fibroblasts are not homogeneous but instead comprise multiple subpopulations with extensive site-to-site and intra-site variations.
\end{abstract}

Keywords: periodontal ligament, fibroblasts, collagen, Alkaline phosphatase

Cite This Article: Dr. A. Archana, Dr. Venkata Srikanth, Dr. Sasireka, Dr. Bobby Kurien, and Dr. Ebenezer, "Fibroblast Heterogeneity in Periodontium - a Review." International Journal of Dental Sciences and Research, vol. 2, no. 3 (2014): 50-54. doi: 10.12691/ijdsr-2-3-1.

\section{Introduction}

\subsection{Background}

Fibroblasts are the predominant cells of the periodontal ligament (PL) and have important roles in the development, function, and regeneration of the tooth support apparatus. Biological processes initiated during the formation of the PL contribute to the long-lasting homeostasic properties exhibited by PL fibroblast populations Over the past decade there has been increasing support for the hypothesis that PDL is directly involved in periodontal repair processes. The formation of the PL is likely controlled by epithelial-mesenchymal and epithelial hard tissue interactions, but the actual mechanisms that contribute to the development of cellular lineages in the PL are unknown.

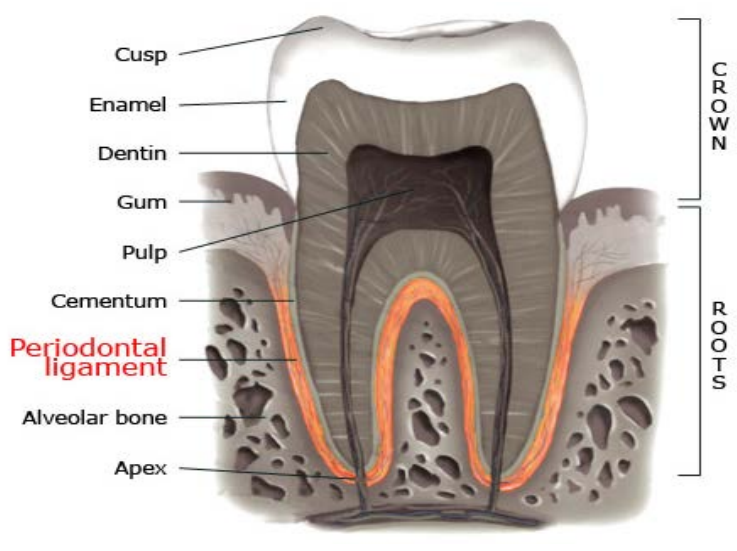

Figure 1. Macroscopic tooth structure

\subsection{Fibroblast Heterogeneity in Systemic tissues}

Morphologic differences were identified in a fetal lung fibroblast line, WI - 38, has been shown to be heterogeneous in cell shape, nuclear morphology, and distribution of organelles

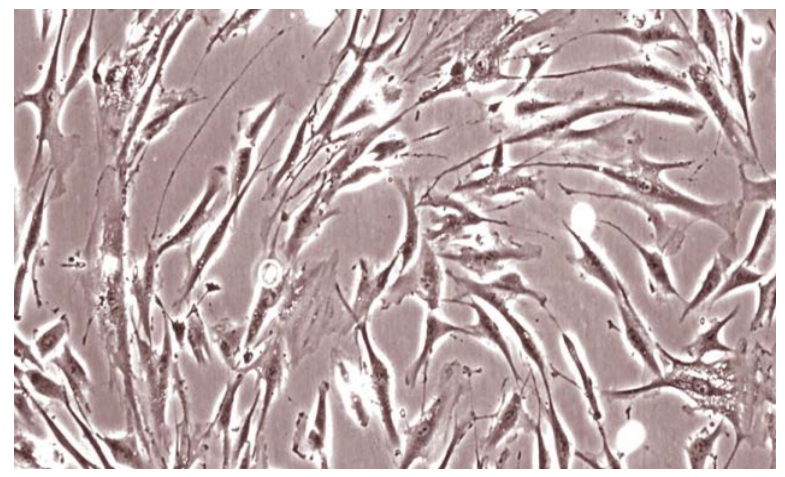

Figure 2. Ultramicroscopic periodontal ligament fibres

\subsubsection{Collagen Production}

Human skin fibroblast clones have been shown to be different in collagen production as much as 3-fold. Studies by Mollenham and Bay reuther observed 3 distinct fibroblast cell types with respect to collagen production. Fibroblasts termed FI produced very little Type I and type III collagen. FII cells synthesized slightly more collagen and large quantities were made by FIII fibroblasts.

\subsubsection{Expression of Diverse inTermediate Filament}

(Gabbioani et al). The concept of the myofibroblasts were distinguished from other fibroblasts by their expression of $\alpha$ smooth muscle actin and/ or desmin 
whereas typical fibroblasts express only vimentin, a high percentage of fibroblast in granulation issue acquire $\alpha$ smooth muscle actin becoming contractile and thus promoting wound closure. IFN- $\gamma$ inhibits fibroblast expression of $\alpha$ smooth muscle actin and reduce retractile conditions and hypertrophic scars. The observation that not all fibroblasts in the wound microenvironment acquire a myofibroblast phenotype supports the concept of fibroblast heterogeneity in a single anatomic site.

\subsubsection{Fluorescence Activated Cell Sorting (FACS)}

strategy employs antibodies or ligands to cell surface molecules

FACS has been used to separate subpopulation on the basis of

- Thy 1 ,

- Class II MHC antigen,

- collagen receptors,

- Clq receptors and

- estrogens receptors.

\section{Evidence of Fibroblast Heterogeneity in Periodontal Tissues}

Two type of periodontal fibroblast defined by tissue localization.

The Gingival Fibroblast (GF) construct the soft connective tissue which surround the alveolar bone. These fibroblasts produce and maintain the extra cellular components which provide for the integrity of the tissue.

The PDL fibroblasts (PDLF) produces and maintains the connective tissue attachment which firmly anchors the tooth to the alveolus

\subsection{Major Themes}

One is that fibroblasts from different areas of the periodontium are different, namely PDL and gingival

The second theme is that within each of these specialized tissues there are likely to be multiple subsets of fibroblasts

\subsection{Heterogenetic Features}

Rose et al reported that PDL possessed glycogen pools within cytoplasm with many bands of contractile type of microfilaments. They found no contractile microfilaments in GF

Mariotti \& Cochran reported slight differences in proliferative rates with GF growing more rapidly than PDL fibroblasts. Of greater significance were the distribution of GAGs in the cellular fraction of PDL tissue indicative of fibroblasts heterogeneity

Variations in cell morphology

a. Mass-cultured GF are relatively small and fusiform in shape.

b. GF-C1: cells are large and fat and fusiform

c. GFCS (GF clone 5); cells are large and elongated

d. GF-C6 (GF clone 6); polygonal cells are constantly found in this clone.

e. Mass cultured PF; cells are small and slender in shape.

f. PF-C1 (PF clone 1); cells are large and relatively elongated in shape. g. PF-C3 (PF clone 3); cells are large and trapezoid in shape.

h. PF-C5 (PF clone 5); cells are shorter and smaller in size than their mass culture.

i. Ogata et al showed that GF released significantly large amounts of PGE2 compared to PDLF in response to bradykinin or histamine. They also demonstrated that PDLF were not spindly, had higher growth rates and Alkaline phosphatase activity than GF.

ii. Hassel and Stanek in 1983 described in healthy human gingival, that fibroblasts grown from a single biopsy of the papilla tip displayed differences in size, proliferating rate and replicative lifespan in vitro.

iii. Gingival and PDL fibroblasts heterogeneity in expression of other surface markers such as CD 4, CD 40 and CD 60 has been described

iv. Sommerman et al demonstrated significant differences in protein and collagen production in PDLF vs Gf.

v. Hou \& Yaeger (1993) revealed differences in ECM production by gingival \& PDL fibroblasts, where immunofluorescense analysis revealed weak expression of type I and III collagen by GF and strong staining by PDLF.

\section{Differences Between GF \& PDLF}

\subsection{Differential Gene Expression on GF \& PDLF}

One hundred sixty-three genes were found differentially expressed by at least three-fold between PDLF and GF Genes encoding transmembrane proteins and cytoskeleton - related proteins tended to be up-regulated in PDLF whereas genes encoding cell-cycle regulation proteins and metabolism-related proteins tended to be up-regulated in GF. Periostin was observed to be preferentially expressed in the periosteum and PDL indicating its tissue specificity and potential role in maintaining tissue structure.

i. S100A4 was reported as an extra cellular inhibitor of mineralization and maybe one of the factors responsible for keeping PDL space free of mineralization.

ii. On the other hand GF displayed up to a 13 fold higher constitutive level of CD 40 than PDLF

\subsection{Metabolism Related Genes}

i. In addition significant up regulation of metabolism related genes involved in the synthesis of protein and fatty acids and the processing of nucleic acids in GF compared with PDLF may further corroborate the enhanced GF proliferation compared with PDLF.

ii. There has been evidence that GHF has a significantly greater proliferation rate (Larjava t al 1 989) than PDLF. Therefore the increased expression of cell cycle regulation and metabolism related genes may partly explain enhanced GF proliferation compared with PDLF observed in periodontal wound healing.

\subsection{Cytokines}

IL-8 mRNA was found to be highly expressed in GF compares with PDLF with a differential expression of 85.1 fold. Studies have shown that constitutively high levels of IL-8 are observed in culture human gingival fibroblasts (Kent et 1996) possibly due to 
1) GF is ore engaged to respond to inflammatory stimuli than PDLF

2) neutrophil mediated proinflammatory processes maybe regulated in part by GF in the cytokine network of immunoparticipant cells. (Takashiba 1992)

3) GF may paly a role a role in IL-8 production in the formation of cytokine network (Takigawa et al 1994)

\section{Differences in Growth Characteristics of Fibroblasts in Periodontal Regeneration}

There are specific differences in the growth characteristics of human fibroblasts derived from periodontal ligament and gingiva in culture. (Angelo J. Mariotti 1990) Similar growth conditions likely result after periodontal surgery or in cases of pathological loss of connective tissue. Fibroblasts derived from gingiva became confluent by day 4 whereas fibroblasts from periodontal ligament were not confluent until day 6. An explanation for gingival cells reaching confluence earlier than periodontal ligament fibroblasts, despite similar cell generation times (slope of logarithmic growth curve), may be that gingival cells are larger than periodontal ligament cells.

\subsection{Growth Characteristics}

The synthesis of total protein and collagen over seven days was similar between fibroblasts derived from periodontal ligament and gingiva. Analysis of collagen and noncollagen protein synthesis revealed a greater trend in noncollagen protein synthesis in the GF cultures compared to PDLF cultures. which helped to observe Significant functional differences between gingival connective tissue and periodontal ligament connective tissue.

\subsection{Apoptotic Rate}

In periodontal tissue sections, a significantly reduced apoptotic rate was demonstrated in PDLF compared with GF. In vitro, IGF-1 substantially enhanced cell survival in PDLF compared with GF by the up-regulation of antiapoptotic molecules and the down-regulation of proapoptotic molecules.

\subsection{Healing after GTR}

Healing after guided tissue regeneration (GTR) may be explained by differences in functional activities of gingival and periodontal ligament fibroblasts (C. Giannopoulou and G. Cimasoni) GF and PDLF appeared identical under the SEM. Most ECM components increased the proliferation rate of GF and the biosynthetic activity of PDLF. Epithelial cells increased the proliferation of both GF and PDLF but had no effect on their biosynthetic activity.

\subsection{Flow Cytometry Analysis of GTR Associated Periodontal Cells}

The culture human regenerative cells were found to have a fibroblast like morphology similar to cells derived from normal PDL and gingival. Flow cytometry analysis using a panel of antibodies: Collagen type I, fibronectin and tenascin were expressed by all three cells but not equally or uniformly suggesting the presence of subsets. (L. Kuru et al) . Thus a fibronectin-high subset of cells in the membrane associated and regenerated tissue cultures indicated by elevated fibronectin expression.

\section{Phenotypic Differences in Alkaline Phosphatase Expression}

The heterogeneity of periodontal fibroblastic cells in vivo and in vitro indicates that periodontal cells could express either osteoblastic phenotypes (Kawase T, Nojima N) or gingival fibroblast characteristics (Piche JE 1989). HPLF showed a gradual increase in spontaneous alkaline phosphatase activity between proliferative and confluence states, (Matsuda et al ) The ALP expressed by periodontal ligament fibroblastic cells is clearly lower than ROS $17 / 2.8$ (Alliot-Licht B 1991) but is not null, as on gingival fibroblastic cells (Kawase T).

\subsection{Chemotactic Responses of PDLF and GF to Polypeptide Growth Factors}

It has been hypothesized that competition from gingival fibroblasts may reduce the potential of periodontal regeneration PDL cells and GF exhibited dose-dependent migratory responses when challenged with PDGF, IGF-I, IGF-II, EGF, and TGF-beta. Only PDL cells migrated in a specific dose-dependent manner (F. Nishimura and V. P. Terranova).

\subsection{TGF- $\beta 1$ Expression}

TGF- $\beta 1$ is expressed in developing alveolar bone, PDL, and cementum at all stages of tooth development, notably in osteoblasts, PDL fibroblasts, and cementoblasts near the root apex TGF $\beta$ R-I and -RII were weakly expressed in fibroblasts present in normal human gingival connective and PDL tissues and upregulated in regenerated tissue biopsies Fibroblasts derived from PDL exhibited increased cellular proliferation, levels of alkaline phosphatase, collagen and protein synthesis as well as chemotaxis in response to TGF- $\beta$.

\subsection{Integrin Expression}

Expression of fibronectin receptor ( $\alpha 4 \beta 1)$ higher for GF and PDLF than DF, however all fibroblasts adhered equally well to fibronectin. Expression of $\alpha 3 \beta 1$ fibronectin receptor was equally great for PDLF, but lower for GF. PDL cell expression was lower than GF or DF for $\alpha v \beta 8$ both a fibronectin and laminin receptor.

\section{Periodontal Wound Healing}

Phenotypic differences between gingival and periodontal ligament fibroblasts have also been well documented in terms of the number of parameters of potential relevance to wound healing, morphology and growth potential (Hakkinen and Larjava, 1992)including the synthesis of matrix macromolecules (Hou and Yaeger, 1993) and response to TGF- $\beta$ (Dennison et al, 1994; Mailhot et al, 1995). 


\section{1. 'Fetal-like' Fibroblasts}

With specific reference to wound healing, Bucala et al (1994) have provided evidence that a specific subpopulation of circulating fibroblast-like cells enter the wound site by egress from the vasculature. Oral mucosa appears to be a privileged site in the adult in that it tends to display a 'fetal like' pattern of regenerative and scarless wound healing (Shafer et al, 1974; Walter and Grundy, 1992) The possible persistence of 'fetal-like' fibroblasts in the oral mucosa and their potential contribution to the characteristic mode of wound healing at this site have recently been discussed (Irwin et al, 1994).

\subsection{Papillary Fibroblast}

Irwin et al dissected the lamina propria of attached human gingiva into papillary tips and deeper reticular layers and demonstrated that fibroblast from each layer differ in several ways. Papillary fibroblasts appeared small and spindly proliferated faster in primary culture than those isolated from the reticular layers.

\subsubsection{Response to Migration Stimulating Factor (MSF)}

Papillary gingival fibroblasts were similar to fetal skin fibroblasts in their production of the migration stimulating factor (MSF) in contrast to the reticular fibroblasts which displayed a phenotype similar to adult skin fibroblasts that do not produce MSF. The fetal like gingival papillary fibroblasts may thus contribute to migration beneficial non-scarring wound healing in the oral mucosa.

\subsection{Granulation Tissues Fibroblast}

Granulation tissue is formed in connective tissue during wound healing, chronic inflammation, and certain pathological conditions. Phenotypical and functional heterogeneity in HGF and GTF fibroblasts is well documented with differences in morphology, size, and growth rate.

produce different amounts of extracellular matrix components such as proteoglycans, collagens, and matrix metalloproteinases (MMPs); respond differently to inflammatory cytokines and drugs; and express different amounts of integrin-type surface-receptors (fibronectin is regulated by a $5 \mathrm{~b} 1$ integrin)

It has been postulated that granulation tissue arising from gingival connective tissue does not contribute to the formation of hard tissue essential for periodontal regeneration (Larjava et al)

\section{Fibroblast Heterogeneity Pathogenesis Periodontal Disease}

\subsection{Clonal Selection Hypothesis}

Fibroblast-derived proinflammatory mediators and cytokines such as PGE2, IL-1b, IL- 6, or IL-8 may be directly or indirectly implicated in periodontal tissue destruction by promoting fibrosis, granuloma formation or bone resorption. Irreversible changes have been observed in vitro in various phenotypic characteristics of fibroblasts which may be attributed to a positive selection process which results in the predominance of a certain subset (s) of fibroblasts with a unique proliferative and/or synthetic phenotype. (Korn JH 1992) also known as clonal selection hypothesis, was originally proposed by Ko et al This subpopulation (s) may be characterized by a proinflammatory cytokine secretion profile contributing to the pathogenetic mechanisms of the disease.

\subsection{Phagocytosis}

Under physiologic conditions, fibroblasts degrade the collagen matrix by which they are surrounded primarily by a phagocytic pathway. EM study in rats show that the PDL fibroblasts contain more phagocytosed collagen than gingival fibroblasts (Svoboda ELA 1981) suggesting site specific differences in phagocytic capacity. Cultured PDL fibroblasts appear to synthesize more collagen and fibronectin than gingival fibroblasts. Interestingly fibronectin which coats collagen fibrils in vivo (Pitaru et al 1987) has been proposed initiate phagocytosis by acting as a recognition site for fibroblasts (Mc culloch et al 1990). Since fibroblasts phagocytosed fibronectin beads more rapidly than collagen Type I beads, a higher level of fibronectin in the PDL might result in increased phagocytic activity.

\subsection{Nitric Oxide Release}

Van der Paw et al 2000 studied that PDL fibroblasts respond to very weak forces with the release of Nitric oxide whereas gingival fibroblasts do not. This second messenger molecule has been proposed to play a role in tissue remodelling and maintenance of PDL space. Though the precise action is not known, it might play a role in collagen phagocytosis.

\subsection{Collagen Remodeling}

\subsubsection{Collagen Synthesis}

GF or PF showed positive intracellular staining for both Collagen type I and Fibronectin, and Collagen III and Fibronectin. However, there were variations in fluorescence intensity for CI and Fn, ranging from relatively weak to strongly positive. The greater expression of collagen type I and fibronectin in mass cultures and most clones of PDL fibroblasts, in contrast to gingival fibroblasts under the same culture conditions, suggests that PDL fibroblasts are more active biosynthetically than gingival fibroblasts. This observation is consistent with recent observations that synthetic activity (total protein and collagenase digestible protein) of mass-cultured PDL fibroblast is one and a half to two times greater than that of the similarly passaged gingival fibroblasts (Hokama MM, 1987;) This increased synthetic activity of PDL fibroblasts conforms to the reported rapid turnover of collagen in the PDL tissues (Sodek J).

\subsubsection{Collagenase Activity}

The functional activity of collagenase synthesized by PDL fibroblasts has been found to be less than that of gingival fibroblasts (Sodek J 1975).

\subsection{Effects of Mechanical Force}

Primary fibroblasts derived from human periodontium are more resistant to mechanical load than is porcine 
gingival epithelium cell or gingival fibroblasts cultured from canines (Brunette 1984) An identical pattern of mRNA s encoded for MMPs and TIMPS was found in stretched human gingival and PDL fibroblasts. However m RNA s encoding for cellular integrin subunits were different (Bolcato-Bellemin et al 2000).

\subsubsection{Fibroblast Heterogeneity of Signal Transduction Mechanisms to Complement-C1q}

C1q may participate in the compositional change of oral granulation tissues by differentially regulating activities of specific fibroblast subsets. C1q influences fibroblast growth, shape, attachment, and movement. Present studies show that the $\mathrm{cC} 1 \mathrm{qR}$ and $\mathrm{gC1qR}$ fibroblast subsets responded to purified $\mathrm{C} 1 \mathrm{q}$ with a differential generation of $[\mathrm{Ca} 2+] \mathrm{i}$ signals that was coupled to a differential formation of IP3 and redistribution of cPKCs

\section{Conclusion}

The study of fibroblast heterogeneity will make great contributions to the understanding of pathologic processes and wound healing. When the role of various fibroblast subsets is understood manipulation of the response of these cells to injury and environmental challenge may be possible, leading to new and specific therapies for periodontal disease and other connective tissue diseases. Such therapies may include stimulating growth and differentiation receptors on fibroblasts or seeding damaged tissue with fibroblast progenitor cells. Finally, exploring how the fibroblast interacts with host defenses will undoubtedly reveal new information about the pathogenesis of periodontal disease.

\section{References}

[1] Bordin S, Costa LG, Tan X. Fibroblast heterogeneity of signal transduction mechanisms to complement-C1q. Analyses of calcium mobilization, inositol phosphate accumulation, and protein kinases-C redistribution. J Periodontol. 1998 Jun; 69 (6): 642-9.

[2] Bordin S, Page RC, Narayanan AS. Heterogeneity of normal human diploid fibroblasts: isolation and characterization of one phenotype. Science. 1984 Jan 13; 223 (4632): 171-3.

[3] Dahan M, Nawrocki B, Elkaim R, Soell M, Bolcato-Bellemin AL, Birembaut P, Tenenbaum H. Expression of matrix metalloproteinases in healthy and diseased human gingiva. J Clin Periodontol. 2001 Feb; 28 (2): 128-36.
[4] Dongari-Bagtzoglou AI, Ebersole JL. Application of immunoprinting for assessment of fibroblast secretory heterogeneity. J Immunol Methods. 1996 Nov 13; 198 (2): 145-54.

[5] Dongari-Bagtzoglou AI, Warren WD, Berton MT, Ebersole JL CD40 expression by gingival fibroblasts: correlation of phenotype with function. Int Immunol. 1997 Sep; 9 (9): 1233-41.

[6] Fries KM, Blieden T, Looney RJ, Sempowski GD, Silvera MR, Willis RA, Phipps RP. Evidence of fibroblast heterogeneity and the role of fibroblast subpopulations in fibrosis. Clin Immunol Immunopathol. 1994 Sep; 72 (3): 283-92

[7] Giannobile WV, Somerman MJ. Growth and amelogenin-like factors in periodontal wound healing. A systematic review.Ann Periodontol. 2003 Dec; 8 (1): 193-204

[8] Hakkinen L, Larjava H. Characterization of fibroblast clones from periodontal granulation tissue in vitro. J Dent Res. 1992 Dec; 71 (12): 1901-7.

[9] Hassell TM, Stanek EJ 3rd. Evidence that healthy human gingiva contains functionally heterogeneous fibroblast subpopulations. Arch Oral Biol. 1983; 28 (7): 617-25.

[10] Irwin CR, Picardo M, Ellis I, Sloan P, Grey A, McGurk M, Schor SL. Inter- and intra-site heterogeneity in the expression of fetallike phenotypic characteristics by gingival fibroblasts: potential significance for wound healing.J Cell Sci. 1994 May; 107 (Pt 5): 1333-46.

[11] Kent LW, Rahemtulla F, Michalek SM. Interleukin (IL)-1 and Porphyromonas gingivalis lipopolysaccharide stimulation of IL-6 production by fibroblasts derived from healthy or periodontally diseased human gingival tissue.J Periodontol. 1999 Mar; 70 (3): 274-82.

[12] Ko SD, Page RC, Narayanan AS. Fibroblast heterogeneity and prostaglandin regulation of subpopulations.Proc Natl Acad Sci U S A. 1977 Aug; 74 (8): 3429-32.

[13] Larjava H, Heino J, Krusius T, Vuorio E, Tammi M. The small dermatan sulphate proteoglycans synthesized by fibroblasts derived from skin, synovium and gingiva show tissue-related heterogeneity. Biochem J. 1988 Nov 15; 256 (1): 35-40.

[14] Layman DL, Diedrich DL. Growth inhibitory effects of endotoxins from Bacteroides gingivalis and intermedius on human gingival fibroblasts in vitro. J Periodontol. 1987 Jun; 58 (6): 38792.

[15] McCulloch CA, Bordin S. Role of fibroblast subpopulations in periodontal physiology and pathology.J Periodontal Res. 1991 May; 26 (3 Pt 1): 144-54. Review.

[16] McCulloch CA, Knowles G. Discrimination of two fibroblast progenitor populations in early explant cultures of hamster gingiva.Cell Tissue Res. 1991 Apr; 264 (1): 87-94.

[17] McKeown M, Knowles G, McCulloch CA. Role of the cellular attachment domain of fibronectin in the phagocytosis of beads by human gingival fibroblasts in vitro. Cell Tissue Res. 1990 Dec; 262 (3): 523-30.

[18] Seymour RA, Thomason JM, Ellis JS. The pathogenesis of druginduced gingival overgrowth. J Clin Periodontol. 1996 Mar; 23 (3 Pt 1): 165-75

[19] Stabellini G, Calastrini C, Mariani G, Gioia M, Caramelli E, Extracellular glycosaminoglycan changes in healthy and overgrown gingiva fibroblasts after cyclosporin A and cytokine treatments.J Oral Pathol Med. 2004 Jul; 33 (6): 346-53.

[20] Tipton DA, Stricklin GP, Dabbous MK. Links Fibroblast heterogeneity in collagenolytic response to cyclosporine.J Cell Biochem. 1991 Jun; 46 (2): 152-65. 